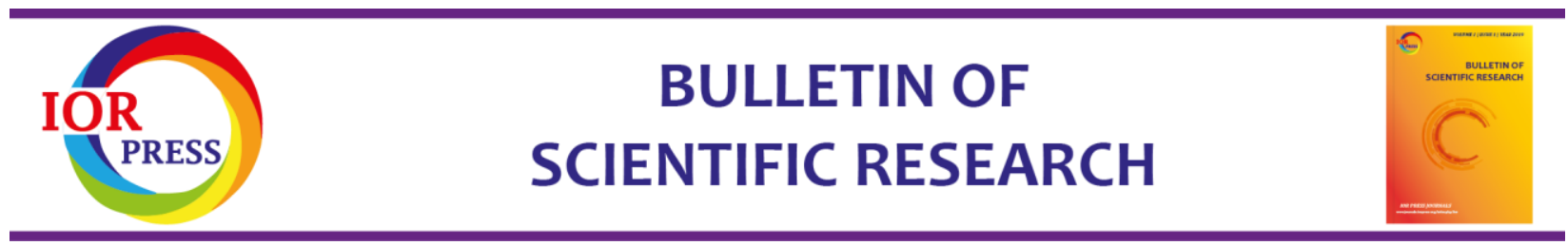

\title{
Design of Rat-Race based Retrodirective Arrays for Backscatter Communications
}

\author{
P. Mahalakshmi a, A. L. Vallikannu ${ }^{\text {a }}$ \\ a Department of ECE Hindustan Institute of Science and Technology, Chennai, Tamil Nadu, India. \\ * Corresponding Author: mahapearl57@gmail.com DOI: https://doi.org/10.34256/bsr2022
}

Received: 28-11-2019

Accepted: 17-01-2020

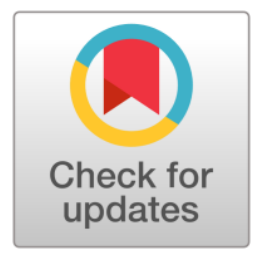

\section{Introduction}

In recent years, interest in wireless communication is increasing due to the development of communication technology. Many RF systems are designed for tracking systems, military communications, radar, and Radiofrequency identification and microwave power transmission [1]. RDA has a simpler structure than a conventional antenna. This high speed and self-tracking process make Retrodirective array useful for RFID and civilian applications by offering automatic maintenance of a high gain link between an interrogator and an RDA.

A Retrodirective array is innately a transponder, as its main function is to wirelessly send a signal response upon being interrogated [2]. Therefore, Retrodirective array is ideal use as passive transponders for a wide range of applications. Battlefield IFF (Identification friend or foe) transponders, and target or project surveillance detection radar for mobile vehicles, are some scenarios which would benefit from an RDA. Retro directive systems can accelerate the detection of targets and reduce the complexity of systems compared with conventional phased arraybased smart antennas.

\section{Rerodirective Technology}

An RDA is a unique type of phased array where beam scanning is achieved automatically and instantaneously [3,4]. It operates by responding toward a source upon incident interrogation with a directivity based on array theory.

\section{A. Van Atta Array}

It consists of a pair of antennas connected by equal lengths of transmission lines creating the symmetric array [2]. The 
received signal creates a phase gradient across the elements in the array based on the incoming signal direction. The received signal at each element is reradiated by its conjugated pair, effectively reversing the phase gradient in the transmitting beam directing it back in the same direction as the originating source. Van atta arrays can operate over a wide bandwidth but require that the incoming wave front and the array itself be planar. Besides, for large arrays, the design of the interweaving feed lines can become intricate.

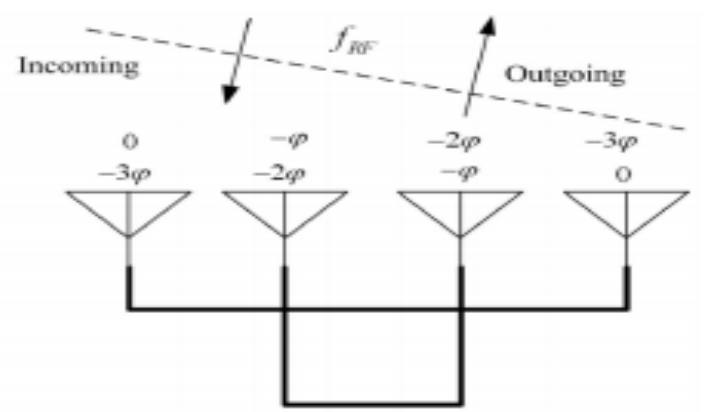

Fig 1. Schematic of Passive van Atta array and the relative phase for transmitted and received signals.

\section{B. Phase Conjugating Array}

The most important method to achieve retro directivity by phase conjugation. This implementation is sometimes known as the heterodyne approach achieves the phase reversal effect in an array at each element using phase conjugating mixers since they are powerhungry devices less used in low - power environments.

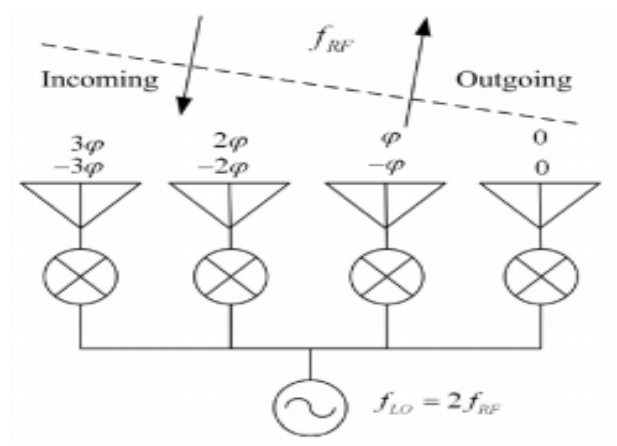

Fig 2. Schematic of phase conjugating or Pon arrays and the relative phase for received and transmitted signals.

\section{Rat-Race Couplers}

The microwave communication technologies are being miniaturized for development in wireless communication systems. The couplers are used for power dividers /combiners with or without phase shift and with equal and unequal power division [5]. The rat-race coupler is a transmission line device in which output appearing at one port depends on the phase of the signals arriving at the port follows different paths.

The coupler is a four-port structure; it can be used as a power combiner or divider. It is a $3 \mathrm{~dB}$ coupler with impedance $\sqrt{ } 2 \mathrm{ZO}$. There are four ports with impedance $\mathrm{ZO}$. It has transmission length of one port has $3 \lambda / 4$ and three with lengths of $\lambda / 4$.

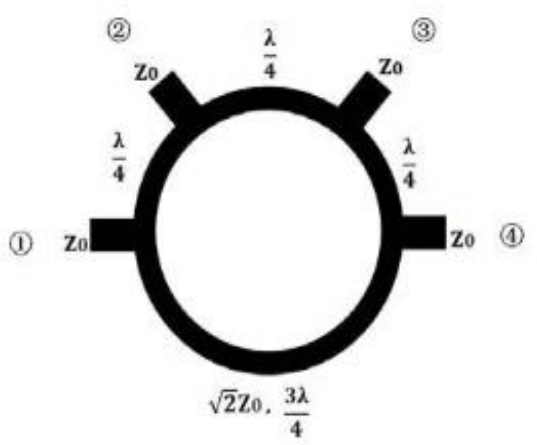

Fig 3. Schematic of Rat-Race Coupler

When port 2 is used as an input port this signal is equally divided into two parts travelling clockwise and anti-clockwise. The distance travelled by the signal from port 2 to port 1 is $5 \lambda / 4$ and the distance travelled from port 2 to port 3 is $\lambda / 4$. Output at port 2 and port 3 are in phase [6]. The signals travelling to port 4 have the path difference of $\lambda / 2$ and due to this $180^{\circ}$ phase difference, no output appears at this port and port being isolated port. If power is applied at port 4 , it divides into two out of phase equal signals between ports 1 and 3 , port 2 being an isolated port. When the device is used as a power combiner, the two inputs are applied at ports 1 and 3 the output appears at port 2 as the summation of these two inputs 
and the difference will appear at port 4. Due to this behavior of the coupler port 2 is called the sum port while port 4 as difference port. This coupler can divide the input power into halves but the output powers are in 0 or 180-degree phase difference.

The S- parameter matrix of the coupler is the following:

$$
\begin{gathered}
{\left[\begin{array}{cccc}
0 & S_{12} & 0 & S_{14} \\
S_{21} & 0 & S_{23} & 0 \\
0 & S_{32} & 0 & S_{34} \\
S_{41} & 0 & S_{43} & 0
\end{array}\right]} \\
\frac{-i}{\sqrt{2}}\left(\begin{array}{cccc}
0 & 1 & 0 & -1 \\
1 & 0 & 1 & 0 \\
0 & 1 & 0 & 1 \\
-1 & 0 & 1 & 0
\end{array}\right)
\end{gathered}
$$

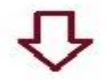

\section{Design and Evaluation}

\section{A. Rat-Race coupler as Retrodirective Array}

Therat-race coupler is Retrodirective if the port 1 and port 2 are connected to the antenna while port 3 and port 4 are terminated with reflection co-efficient. The advantages of using this structure over other techniques such as van atta array are its compatibility and low power consumption. Since Rat -race couplers [7] have a wider bandwidth than other couplers, which is used for high-frequency applications. It is also immune to signal degradation.

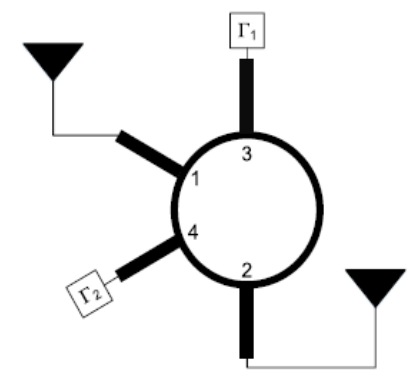

Fig 4. A Rat-Race coupler in which Ports 1 and Ports 2 are connected to antennas and Port 3 and Port 4 is terminated with loads of specific reflection co-efficient.
A passive Retrodirective antenna array overcomes propagation losses and requires no power to scan multiple angles. The phase reversal property can be derived from the scattering matrix. As

$$
[S]=\frac{1}{\sqrt{2}}\left[\begin{array}{cccc}
0 & 0 & e^{-j \frac{\pi}{2}} & e^{-j \frac{\pi}{2}} \\
0 & 0 & e^{-j \frac{3 \pi}{2}} & e^{-j \frac{\pi}{2}} \\
e^{-j \frac{\pi}{2}} & e^{-j \frac{3 \pi}{2}} & 0 & 0 \\
e^{-j \frac{\pi}{2}} & e^{-j \frac{\pi}{2}} & 0 & 0
\end{array}\right] .
$$

The output waves (b3 and b4) at port 3 and port 4 are the input waves (a1 and a2) at port 1 and port 2 are

$$
\begin{gathered}
b_{3}=\frac{1}{\sqrt{2}}\left(a_{1} e^{-\frac{j \pi}{2}}+a_{2} e^{-\frac{j 3 \pi}{2}}\right) \\
b_{4}=\frac{1}{\sqrt{2}}\left(a_{1} e^{-\frac{j \pi}{2}}+a_{2} e^{-\frac{j \pi}{2}}\right) \\
a_{3}=\Gamma_{1} b_{3} \\
a_{4}=\Gamma_{2} b_{4}
\end{gathered}
$$

By setting the condition $\Gamma_{2}=-\Gamma_{1}$, we obtained the Retrodirective condition in ratrace couplers [8]. By comparing the phase difference of the input waves to that of the output waves, the phase of the output is negative concerning input. Thus, the rat-race couplers reverse the incoming phase and act as Retrodirective.

\section{B. Coupler Design}

This proposed model presents a comprehensive description to model Rat-race couplers. Figure 5 is shown the configuration of the Rat-race coupler. The dielectric material is Glass epoxy substrate fr4 $\left(\varepsilon_{r}=4.4\right)$ and the height is $h=1.58 \mathrm{~mm}$ for the operating frequency of $2.4 \mathrm{GHz}$.

- The inner and outer radius of the RatRace coupler is calculated using

$$
R=\frac{3 \lambda_{\mathrm{g}}}{4 \pi}
$$

Inner radius of the ring $r_{1}=\left(r-\frac{w}{2}\right)$ 
outer radius of the ring $r_{2}=\left(r+\frac{w}{2}\right)$

- The wavelength of the coupler is calculated with the velocity of light $\mathrm{C}=3 \times 108 \mathrm{~m} / \mathrm{s}$ and the operating frequency $\mathrm{f}=2.4 \mathrm{Ghz}$.

$$
\begin{gathered}
\lambda_{0}=\frac{c}{f} \\
\lambda_{\mathrm{g}}=\frac{\lambda_{0}}{\sqrt{\varepsilon_{\text {eff } 2}}}
\end{gathered}
$$

- The effective dielectric constant $\varepsilon$ eff1 and reff 2 is calculated using

$$
\varepsilon_{\mathrm{eff}}=\frac{\varepsilon_{\mathrm{r}}+1}{2}+\frac{\varepsilon_{\mathrm{r}}-1}{2}\left(\frac{1}{\sqrt{1+12 \frac{h}{w}}}\right)
$$

- The width for the port input and width for the branches are calculated using the formula

$$
\frac{w}{h}=\frac{8 e^{A}}{e^{2 A}-2} \text { for } \frac{w}{d}<2
$$

where, $A=\frac{z}{60} \sqrt{\frac{\varepsilon_{\mathrm{r}}+1}{2}}+\frac{\varepsilon_{\mathrm{r}}-1}{\varepsilon_{\mathrm{r}}+1}\left(0.23+\frac{0.11}{\varepsilon_{\mathrm{r}}}\right)$

The equivalent circuit of the rat- race couplers of $2.4 \mathrm{GHz}$ is simulated using HFSS EM simulation software [9]. This software is employed to simulate the microstrip patch antenna design. The simulation parameters utilized in the look of rat-race couplers is illustrated in Table 1:

Table1. Parameters for Rat-Race Couplers.

\begin{tabular}{|c|c|}
\hline Parameters & Value (mm) \\
\hline Inner radius (r1) & $17.56 \mathrm{~mm}$ \\
\hline Outer radius (r2) & $16.46 \mathrm{~mm}$ \\
\hline Length of the feed line & $3 \mathrm{~mm}$ \\
\hline Width of the feed line & $9.36 \mathrm{~mm}$ \\
\hline Thickness of the substrate & $1.58 \mathrm{~mm}$ \\
\hline
\end{tabular}

The above figure 6: shows the conventional circuit for the rat- race couplers. For high gain, the intention is to suit the value of the return loss $\left(\mathrm{S}_{11}\right)$ parameter at $-15 \mathrm{~dB}$ at resonant frequency $2.4 \mathrm{GHz}$.

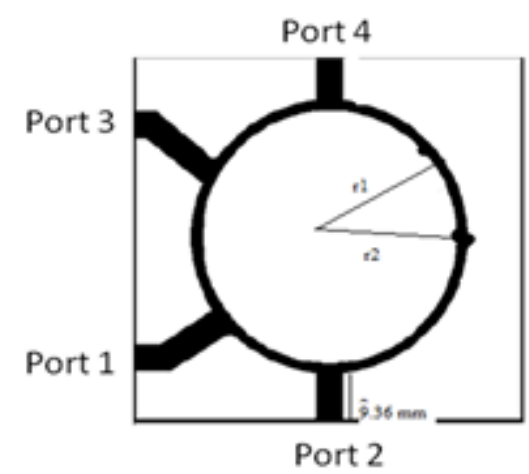

Fig 6. Conventional Rat-Race Coupler

\section{Simulation Results}

The simulated Coupler ring frequency versus magnitude response and phase response are shown in figure 7 (a) and 7 (b) respectively, its return loss is less than $15 \mathrm{~dB}$, insertion loss between $3 \mathrm{~dB}$ to $3.5 \mathrm{~dB}$ and isolation loss less than $20 \mathrm{~dB}$ respectively.

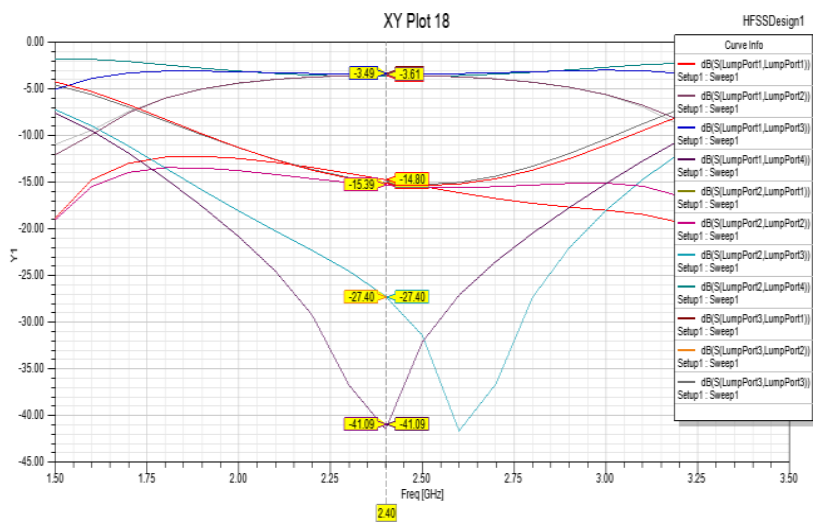

Fig 7(a). Frequency vs. Magnitude response of Hybrid coupler

The phase difference between input and output ports is 179.3 degrees as shown in fig 7(b). The coupler is a four-port device, which divides the signal (port 4) into two equal splits $(3 \mathrm{~dB})$ and in phase between output ports 1 and 2 , port 3 will be isolated [10]. 50-ohm inputs and output ports are excited with three $\lambda \mathrm{g} / 4$ lines and port 2 to port 3 lengths will be $3 \lambda \mathrm{g} / 4$; its impedance is $70.7 \mathrm{ohm}$ as shown in figure 3 . The phase difference between port 4 to port 1 and port 2 is 90 。 


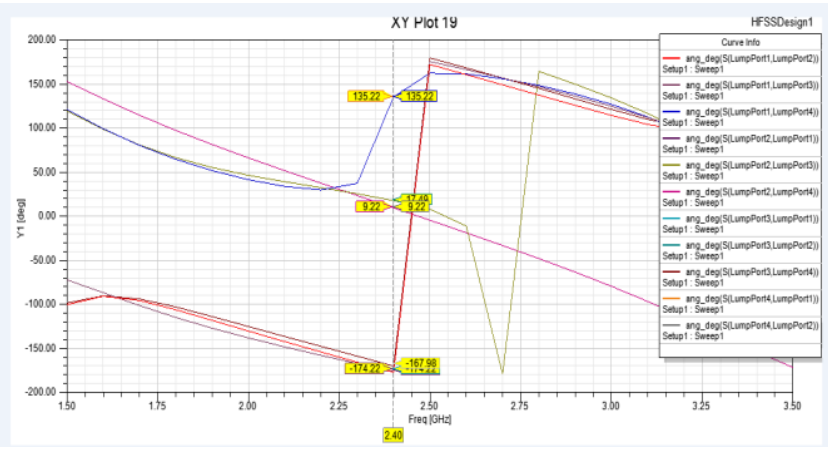

Fig 7(b). Frequency vs. Phase response of Hybrid coupler

\section{Conclusion}

The rat-race coupler is designed and simulated using HFSS EM simulation software. From observing simulation results the return loss and angular phase of s-parameters were obtained. As an extension of the current work, we plan to design Retrodirective RFID mmwave backscatter tags with the rat-race coupler as the feeding network. The feeding network is described clearly how the rat race coupler work in Retrodirective and non-retro-directive mode and simulation and fabrication process is going on. Besides, we plan to include arrays along with rat-race coupler to improve the efficiency of the retro-directive designs and compare their performance.

\section{References}

[1] L.C. Van Atta, “Electromagnetic reflector,” U.S. Patent 2908 002, Oct 6,1959.

[2] E. Nielsen, Square Van Atta Reflector with Conducting Mounting Flame, IEEE Transactions on Antennas and Propagation, 18 (1970) 48-54.

[3] C. Pon, Retrodirective Array Using the Heterodyne Technique, IEEE Transactions on Antennas and Propagation, 12 (1964) 176-180.

[4] David M. Pozer, (2005) Microwave engineering, John Willey and Sons.

[5] Y.-J. Ren, K. Chang, New 5.8-Ghz Circularly Polarized Retrodirective Rectenna Arrays for Wireless Power Transmissions, IEEE Transactions on Microwave Theory and Techniques, 54 (2006) 29702976.

[6] L. Z. Zheng, J. D.Tseng, B. S. Wu, J. M. Li, (2015) Hybrid Type Rat-Race Coupler Designs, In 2015 Asia-Pacific Symposium on Electromagnetic Compatibility (APEMC), IEEE, 724-727.

[7] M. Alhassoun, F. Amato, G. Durgin, A Multi-Modulation Retrodirective Feed Network for Backscatter Communications, In 2017 IEEE 28th Annual International Symposium on Personal, Indoor, and Mobile Radio Communications (PIMRC), IEEE, 1-5. 
[8] Y.C. Chiou, J.T. Kuo, C.H. Chan, New Miniaturize Dual Band Rat-Race Coupler with Microwave CSections, In 2009 IEEE MTT-S International Microwave Symposium Digest, IEEE, 701-704.

[9] S.-N. Hsieh, T.-H. Chu, Linear Retro-Directive Antenna Array Using 90॰Hybrids, IEEE transactions on antennas and propagation, 56 (2008) 1573-1580.

[10] C. Y. Pon, Hybrid -ring directional coupler for arbitrary power divisions, IRE Transactions on Microwave Theory and Techniques, 9(6) 529-535.

Acknowledgement: NIL

Funding: NIL

Conflict of Interest: NIL

About the License: (C) 2020 The Authors. This work is licensed under a Creative Commons Attribution 4.0 International License which permits unrestricted use, provided the original author and source are credited. 